\title{
A Constraint-Based Methodology for Effective Supply Chain Management
}

\author{
A. Lockamy III, CFPIM \\ Florida Agricultural and Mechanical University \\ School of Business and Industry \\ One SBI Plaza, Tallahassee, Florida, USA 32307-5200 \\ (850) $561-2342$
}

Fax: 668-6032

email: lockamy_a@popmail.firn.edu

R. H. Draman, CPIM

School of Business

University of Alabama at Birmingham

1150 Tenth Avenue South

Birmingham, AL, USA 35294-4460

(205) 934-8892

Fax: 934-1845

Email:RDraman@UAB.EDU

\begin{abstract}
This article provides a methodology along with guidelines for employing a "constraint-based" approach for effective supply chain management. The article explores the shortcomings of using a traditional cost management approach in the managing of supply chains. In addition, the article introduces a supply chain management methodology based on achieving a global optimum for the entire chain. Examples are provided on how constraint-based techniques are currently being applied to the management of certain subsets of the supply chain.
\end{abstract}


Guidelines are presented for managing supply chains using a constraint-based approach. Finally, the article ends with a discussion on the implications regarding the use of a constraint-based approach to the management of supply chains along with the need for future research in this area.

\section{Keywords}

Supply-chain management, theory of constraints, cost management

\section{INTRODUCTION}

In an attempt to enhance competitiveness and customer satisfaction, many firms have begun to adopt an integrative approach to the management of its raw materials supply-production-distribution process, commonly referred to as supply chain management. Traditionally, each segment of the supply chain was managed as a stand-alone entity and concentrated on optimizing local objectives without regard to its effect on other parts of the chain. This approach resulted in inter-functional conflicts, unfulfilled company-wide objectives, and reduced levels of customer satisfaction.

With the release of his book The Goal, Dr. Eli Goldratt introduced the world to the impact of system constraints on a manufacturing facility's ability to increase its throughput. Since then, the use of the Theory of Constraints (TOC) by manufacturing firms has provided them with significant business results. Examples include a reduction of manufacturing lead-time from 16 days from 16 hours at Ford Motor Company's Electronics Division, and an increase in plant capacity from 50 to 300 percent at various Avery Dennison plants. This article illustrates how the same "constraint-based" approach, which has been used to improve manufacturing operations, can be applied to the management of supply chains.

\section{PURPOSE}

The purpose of this article is to introduce a methodology along with guidelines for employing a "constraint-based" approach for effective supply chain management. The article explores the shortcomings of using a traditional cost management approach in the managing of supply chains. In addition, the article introduces a supply chain management methodology based on achieving a global optimum for the entire chain. Guidelines are presented for managing supply chains using a constraint-based approach. Finally, the article ends with a discussion on the implications regarding the use of a constraint-based approach to the management of supply chains along with the need for future research in this area. 


\section{COST MANAGEMENT AND SUPPLY CHAINS}

American firms use financial data from their cost management systems to plan and control the operations of their supply chains and, to establish the costs of products and services moving through the supply chains (Johnson, 1992, p. 18). Most of these cost systems are traditional, in that their operating principles and concepts were largely developed prior to 1925 (Johnson and Kaplan, 1987, p. 12). However, some firms are now using the more recently developed ABM system. The next two sections of the article discuss the origins of these systems and drawbacks of using information from them to manage integrated supply chains.

\subsection{Traditional Cost Management}

Not every observer describes traditional cost management in exactly the same way (see Cooper (1988), Johnson and Kaplan (1987), Kaplan (1988), and Sandretto (1985)). Nonetheless, most would agree that traditional cost management is earmarked by the combination of the "managing costs" philosophy once widely practiced by senior U.S. managers (Johnson, 1989, 1990b) and a 1950s vintage product costing methodology (Johnson and Kaplan, 1987, p. 182). This combination often results in managers using misleading cost information in their planning and control decisions.

By the 1980 s, U.S. manufacturers, responding to competitive pressures, had begun to diversified their product offerings. To improve product quality and service, U.S. manufacturers had begun adopting advanced management techniques such as total quality management (TQM), just-in-time (JIT) manufacturing and distribution processes, design for manufacturability (DFM), and flexible manufacturing systems (FMS) (Kaplan,1990). As a consequence, their operating characteristics and cost structures changed dramatically. Overhead replaced direct labor as the major component of manufacturing conversion cost (Eiler, Goletz and Keegan, 1982; Kaplan, 1984; Johnson and Kaplan,1987; Miller and Vollmann, 1985; Reeve, 1989); variable costs were converted to fixed as new manufacturing technologies were purchased (Berliner and Brimson, 1988); inventory, once consider an asset, became a liability (Hayes and Clark, 1986; Kaplan, 1984); and lead time became as important as marketing mix in creating customer value (Blackburn, 1994). This blend of operating factors clashes sharply with the assumptions underlying traditional cost systems. In fact, in this new manufacturing environment, the simple, volume-based overhead allocation schemes of traditional cost systems can not accurately assign the burgeoning factory overhead costs to diverse products. Instead, the traditional cost systems inundate managers with accounting reports that routinely overstated the cost of high-volume, standardized products and understated the costs of lowvolume, customized products.

Clearly, the traditional cost approach is not an adequate framework for managing supply chains. Its most glaring weakness is in the treatment of 
customers. Except for cost, their value perceptions and requirements are ignored. Customers are viewed as uninteresting entities to be cajoled into purchasing the firm's products. Managers, according to the tenets of traditional cost management, should focus exclusively on the internal economics of the supply chain, to minimize its costs. Suppliers, manufacturing methods, and distribution channels should all be selected based on the impact on unit cost. Other aspects of the supply chain strategy are not considered. The traditional cost management approach does not even distinguish between value adding and non-value adding processes or activities for improving the supply chain. The only distinction maintained is between more and less costly activities. The unjustified attending assumption is that all costs add some value that savvy firms can recoup from customers.

\section{A CONSTRAINT-BASED APPROACH TO SUPPLY CHAIN MANAGEMENT}

While the traditional approach relies on what is commonly referred to as 'costbased' data to focus their search for improving the efficiency and productivity of various supply chain activities and associated organizations, TOC takes a different view of supply chains. The Theory of Constraints as defined by the Avraham Y. Goldratt Institute states that "the output of any real system is limited by that system's constraint" (AGI, 1995, p.3). By definition, every supply chain is a system. After all, every supply chain is composed of organizations, each contributing some measure of added value to the end product, that depend on each other for the production and delivery of the end product to the customer. Therefore, the output of any supply chain is determined by that chains constraint, nothing else. The remainder of this paper applies this systems perspective to the control and management of the supply chain.

The first attempt at applying TOC to a supply chain took place in late 1991. Representatives from the four members of an apparel supply chain (see Figure 1) met to shatter their existing paradigm about business and their relationships. The members of this group represented the fiber producer, the textile maker, the apparel producer, and the retailer. Their meetings resulted in the supply chain members realizing that "until the [end] consumer has bought a product, no one in the chain has sold anything" (Schaffner, 1992).

If they are correct, in their recognition that no new money has been added to the supply chain until the end product is sold to the end customer, then, what happens to the chain if it fails to attract and sell more of its products to the end user? Without the addition of new money, how long will the chain, as it is currently structured remain in existence?

This linear representation of their supply chain was sufficient to serve the needs of these paradigm breakers, because their focus was on the apparel manufacturer's 
supply chain. With the exception of the retailer, this chain was composed of firms whose basic product flow is divergent in nature. Divergent or ' $\mathrm{V}$ ' plants are defined by processes that consume a limited number of inputs in their production of a large number of end products. In addition, most of the end products are produced on the same or similar processes (Umble and Srikanth, 1990).

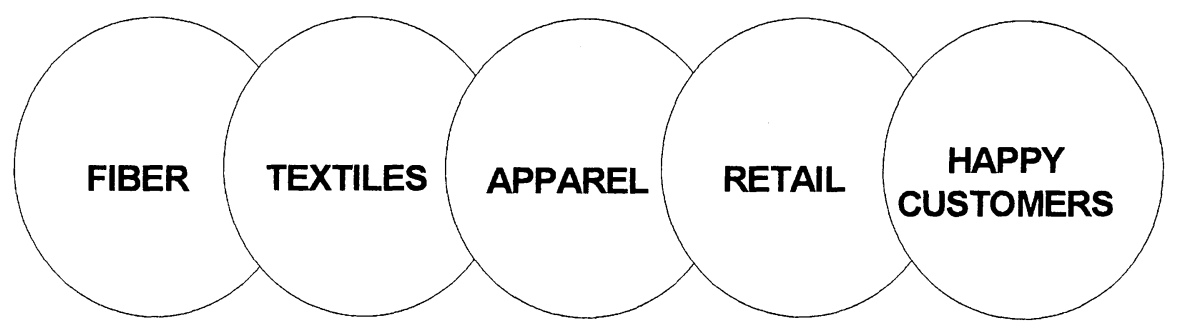

Figure 1 Apparel Supply Chain Members

What about firms whose basic flow is convergent? In that instance, a different, more complex model is needed. Figure 2 presents such a model. To more accurately reflect the realities of a supply chain, we have expanded the linear model to include a number of organizations serving each of the various stages within the chain. The resulting supply chain represents a product or industry that has over 400 retailers selling its product. Each retailer offers their customers a choice from three of the four varieties of product. Each of the regional distributors serves over 100 retailers with product from three of the four end product manufacturers. Each end product manufacturer (EPM) consumes sub assemblies from four of the seven sub-assembly manufacturers (S-AM's) that serve this and other industries. Each of the sub-assembly manufacturers procures materials from the three material finishers (MF's). The material finishers procure their materials from the two basic material processors, who purchase their material from the one raw material extractor.

A word of caution about this model relative to the various 'stage' members from the end manufacturer back through the raw material extractor. We are not assuming or suggesting that the inputs to all the members of any one 'stage' are the same. For example, while all S-AM's purchase material from the three material finishers, the amount or variety consumed by each of the S-AM's is not the same. As one starts to recognize supply chains as a set of interdependent firms that play different 'stages' within different supply chains, one is struck by the webbiness of the various interconnections between the various firms. While our analysis will be limited to this particular supply chain or web, we recognize that each firm, with rare exception, is a participant in a number of different supply chains. 


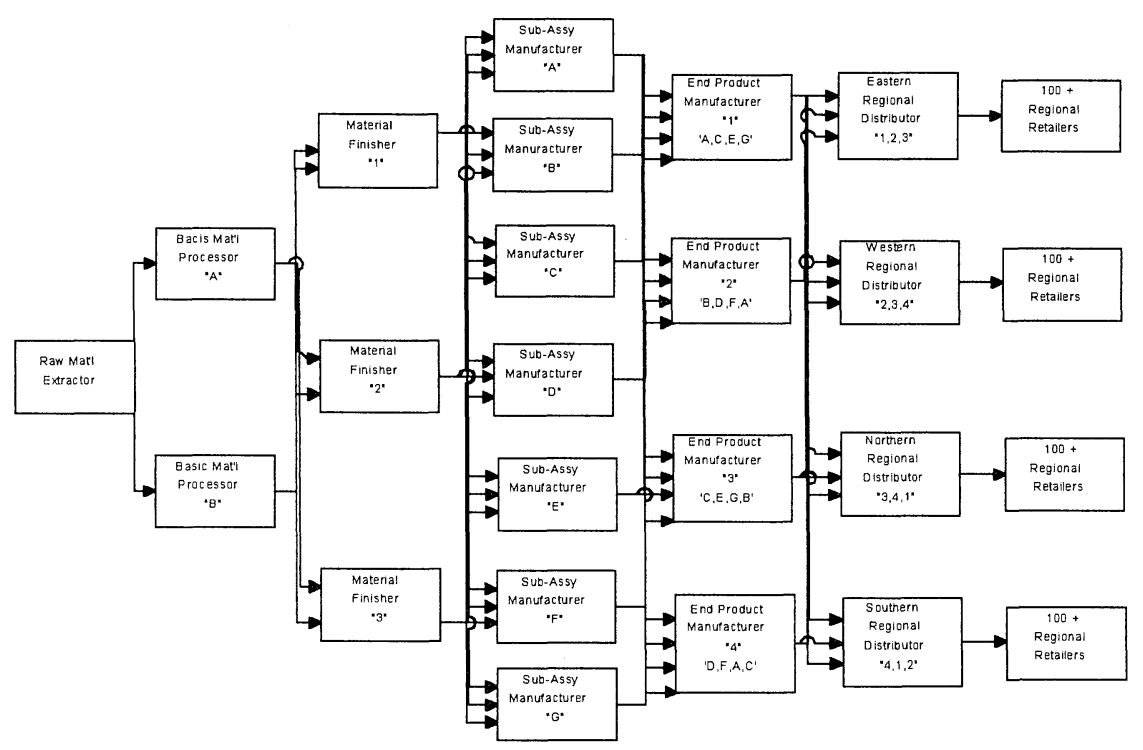

Figure 2 Divergent Supply Chain Model

\subsection{Drum-Buffer-Rope, Buffer Management, and the Supply Chain}

TOC's tools for effectively managing and controlling the flow of products through a system are based upon its five focusing steps. The first step in applying these logistics tools is to 'identify the system's constraint.' In a supply chain this would be translated into 'which stage in the chain is limiting the chain's ability to sell more of its end product to the final customer'? By knowing which element (stage) is limiting a system's output, the system knows where improvement efforts must be focused if output is to be improved.

While finding the constraint within the walls of one organization is relatively simple, locating it within a supply chain is a bit more difficult. The following two guides should help. First, identify the organization (stage) that is usually called upon when there is a need to expedite an order to meet an unexpected demand by the end customer. Second, identify which organization (stage) is holding the most work in process inventory of the product. Finally, remember that when DBR and BM are correctly used, they tend to identify the system's constraint. Therefore, a good guess as to the constraint is all that is needed.

The second of the five focusing steps is to 'exploit the constraint.' Since the chain's output is dependent upon how much the constraint produces, the chain wants to maximize the output of its constraint. In the case of the supply chain, this means that the organization (stage) that is home to the chain's constraint 
must do everything it can not to waste its limited resources relative to its production of the product. Improving the ability of the constraint to produce products, by definition, improves the ability of the system to perform (produce and sell finished goods).

The third step is to 'subordinate all other activities' to the rate of the constraint. Within an organization, this is done so that its excess capacity, capacity that is not needed to meet the system's demand, is identified. In the supply chain the purpose is the same. If this supply chain is being fed at the same rate that the chain's constraint can produce output, than all of the organizations (stages) within the chain can identify any excess capacity relative to that needed to meet the chain's overall demand. This excess capacity can be used by the various organizations (stages) to improve their performance and the performance of the other supply chains.

The fourth stage is to 'elevate the constraint.' If, after going through the first three steps, the system (supply chain) cannot meet the demand being placed on it by the market place, then it must increase the capacity of its constraint. Nothing else will increase the systems ability to produce. The same with the supply chain. If the chain decides it wants to expand (sell more) then it must focus its improvement efforts on elevating the capacity of its constraint. This can be accomplished in a variety of ways: (1) bring on an additional supplier; (2) purchase additional equipment; and, (3) off-load constraint activity to a nonconstraint stage.

The fifth and final step has to do with what must be done if the constraint is elevated. Then, the chain must go back to the beginning and repeat the first three steps. This is done because most elevation efforts result in moving the constraint.

Drum-Buffer-Rope (DBR) and Buffer Management (BM), are TOC's tools for managing the logistical flow of materials through a system. The drum is the constraint schedule. This is also the rate at which the chain is capable of producing output. In the supply chain, this would translate into a drum schedule that is established and continually revised based upon the market demand for the end product. This approach is similar to Wal-Marts daily orders to their manufactures, except when using D-B-R the orders would go to the supply chain's Drum, which may or may not be the end product manufacturer. The Buffer is the amount of time protection positioned in front of the constraint activity that insures it is not disrupted by fluctuations in the preceding stages. Depending on the constraint's location (which stage of the supply chain) and the distance (transportation time) between the various stages in the supply chain, the size of the buffer (amount of time as measured by inventory) could be relatively large. A primary objective of TOC would be to continually reduce the size of the buffers. The Rope serves as the communications link between the critical control points in the chain. These are usually the material release, the constraint, any assembly points where constraint and non-constraint parts are assembled, and shipping. By making sure all of these activities are synchronized, working from the same 
schedule (one derived from the Drum schedule), and that the appropriate buffers are being maintained, the chain's performance is maximized.

At each of the critical control points, TOC places a buffer. The purpose of all buffers is the same, to protect the control point from fluctuations in the preceding activities or stages. By monitoring the buffer content and comparing its actual content to its expected content, the performance of the preceding activities can be determined. The buffer has been sized to allow its expected content to flow through the preceding processes and arrive in place with plenty of time to spare. When this is not occurring, the buffer will signal its manager by revealing a 'hole' where the actual does not reflect the plan. This 'hole' allows the buffer manager to take action before a problem occurs at the critical control point.

\section{CONCLUSIONS}

Based upon the methodology presented above, Table 1 provides guidelines for effective supply chain management. First, individual members of a supply chain must recognize and embrace the global perspective. That is, their organization is a system whose output is controlled by its constraint, and the supply chain[s] they are part of, is also a system whose output is controlled by its constraint. Second, the individual member of the supply chain must adopt DBR and BM. This step will insure that all members are able to maximize their resources and understand how these techniques improve their individual performance. Third, the supply chain members adopt a global point of view regarding the management of the supply chain. The supply chain must be viewed and managed in a holistic manner. Fourth, the element (stage) that is inhibiting optimum supply chain performance (i.e., the constraint) must be identified. Fifth, once the supply chain constraint has been identified, steps must be taken to maximize its capabilities as much as possible. Once the constraint is performing at an optimal level, the sixth step is to synchronize all supply chain activities to the rate of the constraint. This will result in the creation of excess capacity at certain elements (stages) within the supply chain. The seventh step is to develop ways to increase the capability of the constraint within the supply chain. Activities necessary to 'elevate' the constraint will be a function of its nature and location. An effort should be made to continuously improve the performance of the supply chain in relation to its served markets. This process of continuous improvement may result in either the breaking of the original constraint, and/or the shifting of the constraint to another part of the supply chain. When this phenomena occurs, steps 4-7 must be repeated. Finally, in order for effective supply chain management to occur, firms must employ the use of Drum-Buffer-Rope (DBR) and Buffer Management (BM) to ensure optimal supply chain performance. 
Table 1 Guidelines for effective supply chain management

\begin{tabular}{|l|}
\hline 1. Individual supply chain members recognize and embrace a global perspective \\
\hline 2. Individual supply chain members adopt DBR and BM \\
\hline 3. Adopt a global versus local viewpoint of supply chain management \\
\hline 4. Identify the supply chain's limiting factor (constraint) \\
\hline 5. Maximize the output of the supply chain's constraint \\
\hline 6. Synchronize all supply chain activities to the rate of the constraint \\
\hline 7. Develop ways to increase the capability of the supply chain's constraint \\
\hline $\begin{array}{l}\text { 8. Continuously improve the supply chain's performance vis-à-vis its target } \\
\text { markets }\end{array}$ \\
\hline $\begin{array}{l}\text { 9. Employ the use of Drum-Buffer-Rope and Buffer Management within the } \\
\text { supply chain }\end{array}$ \\
\hline
\end{tabular}

Future research is needed to test the robustness of using a constraint-based approach to supply chain management. Empirical research involving supply chains comprised of different supplier, manufacturing, and distribution configurations across a wide range of industries is required to validate and generalize the findings contained in this paper.

\section{REFERENCES}

Available upon request

\section{BIOGRAPHY}

ARCHIE LOCKAMY III, Ph.D., CFPIM is a Professor of Operations Management at the Florida Agricultural and Mechanical University (FAMU). Prior to his academic career, Dr. Lockamy held various engineering and managerial positions with DuPont, Procter and Gamble, and TRW. Dr. Lockamy has published articles in numerous academic journals. He is the co-author of the book Reengineering Performance Measurement: How To Align Systems To Improve Processes, Products and Profits.

REXFORD H. DRAMAN, Ph.D., CPIM is a assistant professor of Operations management at the University of Alabama at Birmingham. Prior to his academic career, Dr. Draman held various managerial positions with Mohawk Tire, Union Metal \& Mfg., Tracor Aerospace, and Eaton Corp.'s AIL Division. Dr. Draman has published and presented numerous articles and papers that focus on the application of the Theory of Constraints. 